\title{
Comparison of Carbon Dioxide Laser With Surgical Blade for Removal of Epulis Fissuratum. A Randomized Clinical Trial
}

\author{
Abbas Karimi ${ }^{1}$, Farhad Sobouti ${ }^{2}$, Sara Torabi ${ }^{3}$, Ali Bakhshandehfard ${ }^{4}$, Armaghan Amirian ${ }^{4}$, Mahsa Shariati $^{*}$, \\ Ehsan Morshedi ${ }^{6}$, Maryam Barati ${ }^{7}$ \\ ${ }^{1}$ Department of Oral and Maxillofacial Surgery, School of Dentistry, Tehran University of Medical Sciences, Tehran, Iran \\ ${ }^{2}$ Orthodontic Department, Dental Faculty, Mazandarn University of Medical Sciences, Sari, Iran \\ ${ }^{3}$ Department of Periodontics, Dental School, Hamadan University of Medical Sciences, Hamadan, Iran \\ ${ }^{4}$ Maxillofacial Surgeon, Private Practice, Tehran, Iran \\ ${ }^{5}$ Department of Oral and Maxillofacial Surgery, Craniomaxillofacial Research Center, Tehran University of Medical \\ Sciences, Tehran, Iran \\ ${ }^{6}$ Department of Prosthodontics, Dental School, Shahed University of Medical Sciences, Tehran , Iran \\ ${ }^{7}$ Laser Application in Medical Sciences Research Center, Shahid Beheshti University of Medical Sciences, Tehran, Iran
}

\author{
*Correspondence to \\ Mahsa Shariat, DDS, MSc.; Laser \\ Research Center of Dentistry, Tehran \\ University of Medical Sciences, \\ Tehran, Iran. \\ Tel: $+98-21-84902473$ \\ Fax: +98-21-84902473 \\ Email: Mahsa.shariati@gmail.com
}

Published online 18 July 2016

\begin{abstract}
Introduction: Epulis fissuratum is often formed as a result of a poor fitting denture. The conventional treatment for this fibrous hyperplastic tissue is to excise it using a scalpel and to close the wound by a continuous or an interrupted suture. The increased utilization of lasers in dentistry also includes the utilization of carbon dioxide (CO2) lasers in place of surgical scalpels in soft tissue surgeries. The objective of this study is to assess the feasibility of utilizing $\mathrm{CO}_{2}$ laser in place of scalpel in surgical treatment of epulis fissuratum.

Methods: In this clinical trial research (IRCT code: IRCT2016071124969N2), 19 patients were selected with nearly symmetrical epulis fissuratums in the anterior part of the jaws. The hyperplastic tissue was evenly divided into two sections in each patient. One section was randomly selected and cut by $\mathrm{CO}_{2}$ laser and the other section by a surgical scalpel. The wound created by the scalpel was closed by appropriate number of interrupted sutures. Surgery duration and bleeding as well as vestibular depth, re-epithelialization and edema in both sections were noted and recorded after 7 and 14 days postoperatively.

Results: The time of surgery and the amount of bleeding during surgery in the laser section was less and the vestibular depth was more than surgical scalpel section $(P<0.05)$. Surgical scalpel wound at day seventh healed significantly better than the section treated by the $\mathrm{CO}_{2}$ laser $(P<0.05)$. Wound in both sections healed similarly on day 14 and no statistical difference was observed. Edema presence was also equal in both sides after 7 th and 14 th following the surgery.

Conclusion: According to the results it could be concluded that the use of $\mathrm{CO}_{2}$ laser may result in less surgery time, less bleeding during surgery, more vestibular depth, better reepithelialization of the wound and less need for suturing. $\mathrm{CO}_{2}$ laser may be a clinically preferred method for surgical treatment of epulis fissuratum.

Keywords: fissuratum; $\mathrm{CO}_{2}$ laser; blade.
\end{abstract}

\section{Introduction}

The epulis fissuratum is a pseudotumor hyperplasia of fibrous connective tissue developing in relation with the flange of denture which is ill-fitting, or partially complete. Although the simple term epulis sometimes is used synonymously for epulis fissuratum, epulis is actually a generic term that can be applied to any tumor of the gingiva or alveolar mucosa. Therefore, some authors have advocated not using this term, preferring to call these lesions inflammatory fibrous hyperplasia or other descriptive names. However, the term epulis fissuratum is still widely used today. ${ }^{1}$

The growth of the tissue in the oral cavity is called "hyperplasia" which is placed over the soft tissue of the alveolar ridge of the vestibular sulcus. Etiologically speaking, it is multifactorial, but many parameters, such as smoking, poor oral hygiene, poor denture fitting, and periodontal diseases are said to be usually associated to it. ${ }^{2}$ The epulis fissuratum typically appears as a single or multiple folds of hyperplastic tissue in the alveolar vestibule. Most often,

Please cite this article as follows: Karimi A, Sobouti F, Torabi S, et al. Comparison of carbon dioxide laser with surgical blade for removal of epulis fissuratum. A randomized clinical trial. J Lasers Med Sci. 2016;7(3):201-204. doi:10.15171/jlms.2016.35. 
there are two folds of tissue, and the flange of the associated denture fits conveniently into the fissure between the folds. The redundant tissue is usually firm and fibrous, although some lesions appear erythematous and ulcerated similar to the appearance of a pyogenic granuloma. The epulis fissuratum most often occurs in middle aged and older adults, as would be expected with a denture-related lesion. It may occur on either the maxilla or mandible while it is more prevalently seen in mandible. The anterior portion of the jaws is affected much more often than the posterior areas and there is a pronounced predilection in women. ${ }^{1}$

There is high prevalence of epulis fissuratum in Iran due to the high amount of inappropriate dentures and consequent severe bone resorption as well as poor oral hygiene. Although epulis fissuratum is the most common lesion in people with complete dentures, malignancies such as squamous cell carcinoma, malignant tumor of salivary glands and metastatic tumors should be considered in each patient. Epulis fissuratum can change over time in to a malignant lesion; therefore, it should be removed as soon as possible. ${ }^{3}$

Carbon dioxide $\left(\mathrm{CO}_{2}\right.$ laser is one of the most powerful lasers suggested as an alternative to the surgical scalpel on soft oral tissues. The $\mathrm{CO}_{2}$ laser was the first laser used for incision and removal of tissues, because of its minimal spot size, good power, high absorption in water and most biological tissues and low risk of infection and scar formation post-operatively. ${ }^{4}$

$\mathrm{CO}_{2}$ laser has been suggested instead of the surgical scalpel for various pre-prosthetic surgeries. Conventional surgical procedures, such as removal of epulis fissuratum with a scalpel causes bleeding and postoperative pain and requires sutures and sometimes tissue grafts. In contrast, minimal post-operational pain, dry excision area and no need for suturing are achieved by using $\mathrm{CO}_{2}$ lasers. ${ }^{5,6}$

In incisional biopsy by scalpel blade, cancer cells can travel through blood stream, which elevate the risk of metastasis, but $\mathrm{CO}_{2}$ laser can seal blood vessels less than 500 micron to decrease this risk, so $\mathrm{CO}_{2}$ laser is useful in malignant lesions removal. ${ }^{7}$

In this study, we compared the use of $\mathrm{CO}_{2}$ laser and the surgical blade for the removal of epulis fissuratum. The main concerns were the duration of surgery, wound healing, re-epithelialization and vestibular depth after lesion removal with each approach.

\section{Methods}

In this clinical trial, 19 candidates of epulis fissuratum removal with anterior symmetrical lesions were selected from Department of Oral and Maxillofacial Surgery at the Faculty of Dentistry of the Tehran University of Medical Sciences. All patients who participated in this study were healthy adults without any remarkable medical problems and no recent history of illicit drug use or medical treatment. The surgeries were similarly done by the same surgeon and same local anesthesia (2 cartridges of $2 \%$ lidocaine with 1:80000 epinephrine via local infiltration).
For each patient one side of the lesion was removed by traditional surgical method using scalpel (supabon \# 15) and the other side was removed by $\mathrm{CO}_{2}$ laser (Smart US20D, Deka, Italy) at wavelength of $10600 \mathrm{~nm}$ The output power of $6.2 \mathrm{~W}$ with frequency of $50 \mathrm{~Hz}$ and pulse duration of 3 $\mathrm{m}$ was used for cutting this lesion. The laser was used in focused mode to generate a $0.7 \mathrm{~mm}$ spot size.

Each procedure was timed from incision to the end of suturing using a chronometer. Therefore, the surgery duration for each side of the jaw was recorded to compare with the other side. The amount of bleeding was measured by the number of sterile gauzes $\left(5 \times 5 \mathrm{~cm}^{2}\right)$ used to prevent bleeding. One sterile gauze utilized, meant no bleeding; two sterile gauzes used was categorized as a low bleeding level and three as "moderate bleeding level." More than three sterile gauzes was identified as "high bleeding level." Surgery was performed on the other side of the lesion with a scalpel using the conventional technique and then closed by interrupted sutures.

Depth of vestibule was measured 7 days and 14 days following the surgery. For each section, vestibular depth was measured in three points (medial, middle and lateral) from the vestibule to the top of the alveolar ridge and the average was calculated for each section with a periodontal probe. Re-epithelialization, as an indicator of wound healing, was measured in 7 th and 14 th postsurgical days. In case of observing concomitant tissue redness and swelling, "no epithelium" was recorded. In case of observing either swelling or redness, the wound was considered in fibroblastic phase and "low epithelium" was recorded and if the wound was in late fibroblastic phase "medium epithelium" and, if in remodeling phase, "complete epithelium" was recorded.

Edema was evaluated and recorded in 7 th and 14th post surgical days by comparing surgery site with adjacent tissues. Each patient was categorized into "edema" and "no edema" group. Duration of the surgeries were analyzed by paired $t$ test and wound healing (re-epithelialization) and edema on days 7 and 14 postoperative as well as bleeding during surgery were analyzed by Wilcoxon signed-rank test. $P$ was less than 0.05 and all the statistical analyses have been done by SPSS ${ }^{\circledR} 11.5$ software.

\section{Results}

A total of 19 patients were enrolled in this study ( 9 female and 10 male) and their ages ranged from 37 to 81 years. The maxilla was involved in 5 cases, the mandible in 14 . The mean duration of surgery was 287.68 seconds in the conventional surgery with the blade and it was $198.68 \mathrm{sec}-$ onds in the surgery using $\mathrm{CO}_{2}$ laser. There was significant difference between the two sides $(P<0.05$; Table 1$)$.

The amount of bleeding on the side done by laser was significantly lower than the side done by surgical blade $(P<0.05$; Table 2).

Mean depth of the vestibule on the medial of the lesion was $4.86 \mathrm{~mm}$ in the conventional surgery with the scalpel and it was $5.11 \mathrm{~mm}$ in the surgery using $\mathrm{CO}_{2}$ laser. There were no significant differences between two sides. While 
Table 1. The Mean \pm SD of Duration of Surgery by Laser and Scalpel Method

\begin{tabular}{lccccc}
\hline Duration of Procedure (s) & Minimum & Maximum & Mean & SD & P (2-tailed) \\
\hline $\mathrm{CO}_{2}$ laser & 50 & 443 & 198.68 & 122.433 & $0.002^{\mathrm{a}}$ \\
Scalpel & 150 & 490 & 278.68 & 103.209 & \\
\hline
\end{tabular}

a Significant difference.

Table 2. The Amount of Bleeding in Laser and Scalpel Method

\begin{tabular}{lccccc}
\hline Bleeding & None & Low & Medium & High & P (2-tailed) \\
\hline Patients in laser group & $8(42.1 \%)$ & $5(26.3 \%)$ & $4(21.1 \%)$ & $2(10.5 \%)$ & $<0.001^{\text {a }}$ \\
Patients in scalpel group & $0(0 \%)$ & $1(5.3 \%)$ & $6(31.6 \%)$ & $12(63.2 \%)$ & \\
\hline
\end{tabular}

a Significant difference.

the mean depth of vestibule in the middle of the lesion in the conventional surgery with scalpel was $4.47 \mathrm{~mm}$ and in the surgery with laser was $5.32 \mathrm{~mm}$ which demonstrated significant difference between the 2 sides $(P<0.05)$. Likewise, the mean depth of vestibule on the lateral of the lesion was $5.05 \mathrm{~mm}$ in the conventional surgery with blade and it was $5.84 \mathrm{~mm}$ in the surgery with $\mathrm{CO}_{2}$ laser which showed significant difference between two sides $(P<0.05$; Table 3).

At postoperative seventh day, there was no significant difference between edema of the two groups, re-epithelialization was better in the side done by scalpel blade than the side done by laser $(P<0.05)$. Likewise, at the 14 days postoperatively there were no significant differences between edema and re-epithelialization of the groups (Tables 4 and 5).

The measure regarding presence of edema in 7 th and 14 th postoperative days showed no significant difference among the groups.

\section{Discussion}

The purpose of this study was to compare the use of $\mathrm{CO}_{2}$ laser with surgical scalpel to remove epulis fissuratum.
Present study results showed lower amount of bleeding and shortest duration of surgery and higher vestibular depth with $\mathrm{CO}_{2}$ laser. The wound healing on day 7 postoperative was significantly better in conventional surgery with scalpel blade while on 14th postoperative day there was no significant difference in wound healing between the two groups.

Tamarit-Borrás et al recommended the output power of $5-10 \mathrm{~W}$ is suitable for removing this lesion, because more than this amount may destroy the tissue. ${ }^{2}$ In the current study in order to prevent tissue destruction and with consideration to the manufacture instructions, the beam of 6.2 W was used. de Arruda Paes-Junior et al and also Suter et al reported significant low amount of bleeding in excisional biopsy by $\mathrm{CO}_{2}$ laser without suturing. ${ }^{8,9}$ Our findings showed that the bleeding was lower with $\mathrm{CO}_{2}$ laser compared to surgery with scalpel. Fisher and Frame reported reduction in vestibular depth in fibro epithelial hyperplasia surgery. ${ }^{10}$ In the present study; at 14 th day postoperatively the depth of vestibule was significantly higher in the surgery with $\mathrm{CO}_{2}$ laser compared to conventional surgery with blade. Rezvan and Mahmoudhashemi reported better wound healing on the side done by laser

Table 3. Mean Depth of the Vestibule in Laser and Scalpel Method

\begin{tabular}{|c|c|c|c|c|c|}
\hline Depth of Vestibule ( $\mathrm{mm})$ & Minimum & Maximum & Mean & SD & $P$ (2-tailed) \\
\hline \multicolumn{6}{|l|}{ Medial } \\
\hline $\mathrm{CO}_{2}$ laser & 3 & 8 & 5.11 & 1.823 & \multirow[t]{2}{*}{0.076} \\
\hline Scalpel & 3 & 8 & 4.86 & 1.729 & \\
\hline \multicolumn{6}{|l|}{ Middle } \\
\hline $\mathrm{CO}_{2}$ laser & 3 & 9 & 5.32 & 1.857 & \multirow[t]{2}{*}{$0.018^{\mathrm{a}}$} \\
\hline Scalpel & 2 & 8 & 4.47 & 1.808 & \\
\hline \multicolumn{6}{|l|}{ Lateral } \\
\hline $\mathrm{CO}_{2}$ laser & 3 & 9 & 5.84 & 1.803 & \multirow[t]{2}{*}{$0.005^{\mathrm{a}}$} \\
\hline Scalpel & 3 & 8 & 5.05 & 1.682 & \\
\hline
\end{tabular}

a Significant difference.

Table 4. Assessment of Re-epithelialization in Laser and Scalpel Method

\begin{tabular}{|c|c|c|c|c|c|}
\hline Re-epithelialization & No Epithelium & Low Epithelium & Medium Epithelium & Complete Epithelium & $P$ (2-tailed) \\
\hline \multicolumn{6}{|l|}{7 day post-op } \\
\hline $\mathrm{CO}_{2}$ laser & $5(26.3 \%)$ & $10(52.6 \%)$ & $4(21.1 \%)$ & $0(0 \%)$ & $0.021^{\mathrm{a}}$ \\
\hline Scalpel & $0(0 \%)$ & $12(63.2 \%)$ & $7(36.8 \%)$ & $0(0 \%)$ & \\
\hline \multicolumn{6}{|l|}{14 day post-op } \\
\hline $\mathrm{CO}_{2}$ laser & $0(0 \%)$ & $0(0 \%)$ & $8(42.1 \%)$ & $11(57.9 \%)$ & 0.180 \\
\hline Scalpel & $0(0 \%)$ & $0(0 \%)$ & $5(26.3 \%)$ & $14(73.7 \%)$ & \\
\hline
\end{tabular}

a Significant difference. 
Table 5. Assessment of Edema in Laser and Scalpel Method

\begin{tabular}{lccc}
\hline Edema & Present & Absent & $\boldsymbol{P}$ (2-tailed) \\
\hline 7 day post-op & & & \\
$\mathrm{CO}_{2}$ laser & $5(26.3 \%)$ & $14(73.7 \%)$ & 0.000 \\
Scalpel & $5(26.3 \%)$ & $14(73.7 \%)$ & \\
14 day post-op & & & \\
$\mathrm{CO}_{2}$ laser & $0(0 \%)$ & $19(100 \%)$ & 0.000 \\
Scalpel & $0(0 \%)$ & $19(100 \%)$ & \\
\hline
\end{tabular}

compare to the side done by blade in 7 and 14 day after the surgery ${ }^{11}$ but in our study, wound healing was significantly better on the side done by scalpel compared to the side done by laser. This might be due to the thermal damage caused by the use of laser. Paes-Junior and Niccoli-Filho also reported delay in healing in surgery with $\mathrm{CO}_{2}$ laser. ${ }^{12}$ However, in our study there was no significant difference in re-epithelialization or wound healing between the 2 sides, 14 days after the procedures. Kauvar et al histologically demonstrated that the $\mathrm{CO}_{2}$ laser, in both the pulse and continuous modes allowed superficial ablation with minimal thermal damage. ${ }^{13}$ Some studies like Dobry et al study also affirmed that the pulse mode caused less thermal damage,but that a longer actuation time was needed with the tissue. ${ }^{14}$ In our study, we used pulse mode.

As the present study has revealed, among other vital and extra uses of $\mathrm{CO}_{2}$ laser, one may refer to their cutting precision, reducing post-op patients' complaints, including pain, edema, and infection; and the non-contact technique, which is unique. Considering the lesion size, it seems remarkable in our case both intra-op, and postop wise, there was no pain, which implies the $\mathrm{CO}_{2}$ laser advantage, which numerous researches have also pointed out. Due to blood and lymphatic vessel sealing, as well as with the prevention of extravasations of the pain and inflammation related fluids, Pogrel et al have attributed this fact to $\mathrm{CO}_{2}$ laser use. ${ }^{15}$ In addition, the irradiation by laser may bring about sealing of the nerve ending in the contact area. The denaturalized collagen layer which is formed on the surgical wound surface serves to isolate from the fluids of the oral cavity. ${ }^{16}$ It took about 20 days for the healing process to complete with no scarring, and with the integrity of the anatomic sulcus.

\section{Conclusion}

The use of $\mathrm{CO}_{2}$ laser for the surgical removal of epulis lesions has resulted in many significant improvements including convenient mucosa removal, better wound healing and re-epithelialization, no bleeding or need for sutures, minimal postoperative pain and edema and less vestibular depth decreased compared to conventional surgery with scalpel blade, more studies are needed to reveal other advantages and disadvantages of this approach.

\section{Ethical Considerations}

This study was approved by both ethics committee of Tehran University of Medical Sciences and its IRCT code is IRCT2016071124969N2. Informed consent was obtained from all participants prior to the study.

\section{Conflict of Interests}

The authors declare that they have no conflict of interests

\section{References}

1. Neville BW, Damm DD, Allen MA. Oral and Maxillofacial Pathology. 3rd ed. New York: W B Saunders; 2008.

2. Tamarit-Borrás M, Delgado-Molina E, Berini-Aytés L, Gay-Escoda C. Removal of hyperplastic lesions of the oral cavity. A retrospective study of 128 cases. Med Oral Patol Oral Cir Buca 2005;10(2):151-162.

3. Sahebjami M, Mansourian A, Momenbeitollahi J, Tohidastekrad Z, Bayatmovahed S, Jafari S. Oral Medicin. 1sted. Tehran: Tehran University of Medical Sciences; 2007.

4. Truschnegg A, Acham S, Kiefer BA, Jakse N, Beham A. Epluis:a study of 92 cases with special emphasis on histopathological diagnosis and associated cilinical data. Clin Oral Investig. 2015;23(3):113-120.

5. Mogdas-Vegara A, HUeto-Madrid JA, Chimenos-Kustner E, Bescos-Atin C. Oral leukoplakia treatment with the carbon dioxide laser: A systematic review of the literature. J Craniomaxillofac Surg. 2016;44(4):331-6.

6. Monterio LS, Mouzinho J, Azevedo A, Camara MI, Martins MA, La Fuente JM. Treatment of epulis fissuratum with carbon dioxide laser in a patient with antithrombotic medication. Braz Dent J. 2012;23(1):77-81.

7. Kusukawa J, Suefuji Y, Ryu F, Noguchi R, Iwamoto O, Kameyama T. Dissemination of cancer cells into circulation occurs by incisional biopsy of oral squamous cell carcinoma. J Oral Pathol Med. 2000;29(7):303-307.

8. de Arruda Paes-Junior TJ, Cavalcanti SC, Nascimento DF, et al. $\mathrm{CO}(2)$ Laser Surgery and Prosthetic Management for the Treatment of Epulis Fissuratum. ISRN Dent. 2011;2011:282361. doi:10.5402/2011/282361.

9. Suter VG, Altermatt HJ, Sendi P, Mettraux G, Bornstein MM. $\mathrm{CO}_{2}$ and diode laser for excisional biopsies of oral mucosal lesions. A pilot study evaluating clinical and histopathological parameters. Schweiz Monatsschr Zahnmed. 2010;120(8):664-671.

10. Fisher SE, Frame JW. The effects of the carbon dioxide surgical laser on oral tissues. Br J Oral Maxillofac Surg. 1984;22(6):414-425.

11. Rezvan B, Mahmoudhashemi H. Comparative survey on carbon dioxide laser and surgical scalpel removal of epulis fissuratum. The Journal of Oral Laser Applications. 2007;7(3):187-190.

12. Paes-Junior TJ, Niccoli-Filho W. Clinical comparison between conventional suture and vaporization with carbon dioxide laser in rat's skin. J Clin Laser Med Surg. 2001;19(6): 319-324.

13. Kauvar AN, Waldorf HA, Geronemus RG. A histopathological comparison of "char-free" carbon dioxide lasers. Dermatol Surg. 1996;22(4):343-348.

14. Dobry MM, Padilla RS, Pennino RP, Hunt WC. Carbon dioxide laser vaporization: relationship of scar formation to power density. J Invest Dermatol. 1989;93(1):75-77.

15. Pogrel MA, Yen CK, Hansen LS. A comparison of carbon dioxide laser, liquid nitrogen cryosurgery, and scalpel wounds in healing. Oral Surg Oral Med Oral Pathol. 1990; 69(3):269-273.

16. Pogrel MA. The carbon dioxide laser in soft tissue preprosthetic surgery. J Prosthet Dent. 1989;61(2):203-208. 